\title{
Robust Switching Control Systems with Input Delay
}

\author{
Vojislav Filipovic \\ Faculty of Mechanical Engineering, University of Kragujevac, \\ PO Box 126, 15300 Loznica, Serbia, \\ v.filipovic@open.telekom.rs
}

\begin{abstract}
This paper uses the concept of multiple models and the concept of switching controllers. The analog part of the system is described by a finite set of continuous-time models with input delays. The continuous-time models include unmodeled dynamics in the form of affine matrix family. By using suitable transformation, the models with input delay are converted into delay free models. Then, by using the LMI tool, the class of robust LQ controllers with the prescribed degree of stability is proposed and for every controller the upper bound for the performance index is given. The switching logic is based on the selection of minimal upper bound from the collection of upper bounds. Finally, it is shown by means of performance dominant conditions, that the resulting closed-loop is stable.
\end{abstract}

Keywords: Multiple models, Input delay, LMI inequalities, Switching rule, Stability

\section{Introduction}

Hybrid systems describe the interaction between software, modeled by finite-state systems such as finite-state machines, and the physical world described by differential equations. The several key verification and control synthesis results for hybrid systems, guided by the concept of bisimulation, are outlined in the reference [1]. From the classical control theory point of view the hybrid systems can be considered as a switching control between analog feedback loops [2]. There are numerous applications of switching systems. For example, the reference [3] presents a vision based cart-inverted pendulum system under a hybrid feedback configuration. In [4] was considered unicycle and constrained pendulum as examples for switched control systems. The reference [5] presents impedance control of a robot manipulator using hybrid control.

Time-delay occurs in many dynamical systems such as biological systems, process industry, and long transmission lines in pneumatic systems, hydraulic systems, and electrical networks. It is frequently a source of the generation of oscillation, instability and poor performance. The effect of delay on the stability properties of dynamical systems is considered in [6]. Design of feedback control law for time-delay systems, based on dynamic programming, is presented in [7].

In this paper we use predictor like techniques for systems with input delay. Such techniques are considered in [8] and [9]. By using a suitable transformation the original problem can be described in the form of delay free system.
A specific class of problems (robust switching control in the presence of input saturation and switching stochastic nonlinear systems) is considered in [11] and [12].

In this paper we describe input delay systems using the multiple-model concept. The model includes unmodeled dynamics in the form of affine matrix family. By using the LMI (linear matrix inequalities) tool [13] the robust hybrid LQ controller with the prescribed degree of stability is proposed. It is formally proved that such a switching closed-loop system is stable.

\section{System Description by Multiple Models}

We assume that the process model is a member of admissible process models

$$
F=\bigcup_{p \in P} F_{p} \text {, }
$$

where $P$ is matrix index set which represents the range of parametric uncertainty so that for each fixed $p \in P$ the subfamily $F_{p}$ accounts for unmodeled dynamics. Usually $P$ is the compact subset of finite-dimensional normed vector space. The process with input delay will be described in the following form

$$
\begin{aligned}
& \dot{x}(t)=A_{p}(q) x(t)+\sum_{i=0}^{r} B_{p i} u\left(t-h_{i}\right), \\
& h_{0}=0 \text {, } \\
& A_{p}(q)=A_{p 0}+\sum_{i=1}^{l} q_{p i} A_{p i} \quad, \quad q_{p} \in Q_{1} \subset R^{l}
\end{aligned}
$$

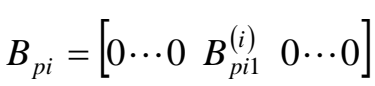


$u^{T}(t)=\left\lfloor u^{0^{T}}(t) u^{(1)^{T}}(t) \cdots u^{(r)^{T}}(t)\right\rfloor$

$p=1,2, . ., s$

where $x \in R^{n}, u \in R^{m}$ and $A_{p}(q)$, and $B_{p i}$ are $n x n$ and $n x m$ matrices respectively. The $h_{i}(i=1,2, . ., r)$ is the input delay. The dimensions of vectors $u^{(i)}(t)(i=1,2, . ., r)$ correspond to the dimensions of matrices $B_{p i 1}^{(i)}(i=1,2, . ., r)$. The initial condition for system (2) is $x\left(t_{0}\right)$ where $t_{0} \geq 0$ is arbitrary finite and known quantity.

Remark 1: In relation (2) the matrices

$A_{p i}, \quad p=1,2, . ., s, \quad i=0,1, . ., r$

are known and

$\left|q_{p i}\right|<\gamma_{p}, \gamma_{p}>0$ for $p=1,2, . ., s$

The maximum $\gamma_{p}=\gamma_{p \text {, max }}$, for which robust stability of affine family of matrices is provided for $\forall \gamma_{p}<\gamma_{p \text {,max }}$, is known as the stability radius.

Relation (2) describes the continuous part of the system. The event driven part can be described in the following form

$p^{+}(t)=\varphi_{1}(p(t), \sigma(t))$,

where $p(t)$ is a discrete event variable, $\sigma(t)$ is a discrete input and $\varphi_{1}(\cdot, \cdot)$ is a function which describes the behavior of $p(t)$. It is important to note that

$$
p^{+}(t)=p\left(t_{n+1}\right), \mathrm{p}(t)=p\left(t_{n}\right), t_{n}<t_{n+1},
$$

A specific form of switching sequence will be described in the next part of the paper.

\section{Switching Control Systems}

For complex processes the regulation problem can be solved, by the family of controllers from [14]

$$
\left\{C_{q^{c}}: q^{c} \in D\right\} \text {, }
$$

where $D$ is the index set. It is supposed that the family is sufficiently rich so that every admissible process model can be stabilized by controller $C_{q^{c}}$ for some index $q^{c} \in D$. This paper will consider the case
$F=D$,

The supervisor (4) and (5), in this paper, is different in comparison with the supervisor described in [2] . Here, the main ingredient of supervisor is the index of performance of feedback control systems.

A transformation will now be formulated, for system (2), which puts system (2) in the finite dimensional form. It means that the transformed system will be described by an ordinary differential equation. That will be given in the following lemma.

Lemma 1. System (2), by next transformation

$$
y(t)=x(t)+\int_{t-h_{0}}^{t} e^{-A_{p}(q)(s-t)} e^{-A_{p}(q) h_{0}} B_{p 0} u(s) d s+
$$

$\int_{t-h_{1}}^{t} e^{-A_{p}(q)(s-t)} e^{-A_{p}(q) h_{1}} B_{p 1} u(s) d s+\cdots+$

$+\int_{t-h_{r}}^{t} e^{-A_{p}(q)(s-t)} e^{-A_{p}(q) h_{r}} B_{p r} u(s) d s$

obtains the form

$$
\begin{aligned}
& \dot{y}(t)=A_{p}(q) y(t)+\sum_{i=0}^{r} e^{-A_{p}(q) h_{i}} B_{p i} u(t) \\
& p=1,2, . ., s, h_{0}=0
\end{aligned}
$$

Proof: By differentiation of the transformation relation one can get

$$
\begin{aligned}
& \dot{y}(t)=\dot{x}(t)+e^{-A_{p}(q) h_{0}} B_{p 0} u(t)-B_{p 0} u\left(t-h_{0}\right) \\
& +A_{p}(q) \int_{t-k_{0}}^{t} e^{-A_{p}(q)(s-t)} e^{-A_{p}(q) h_{0}} B_{p 0} u(s) d s+ \\
& +e^{-A_{p}(q) h_{r}} B_{p r} u(t)-B_{p r} u\left(t-h_{r}\right)+ \\
& +A_{p}(q) \int_{t-h_{r}}^{t} e^{-A_{p}(q)(s-t)} e^{-A_{p}(q) h_{r}} B_{p r} u(s) d s
\end{aligned}
$$

Using relations (7) and (2) we obtain

$$
\begin{aligned}
& \dot{y}(t)=A_{p}(q) x(t)+\sum_{i=0}^{r} B_{p i} u\left(t-h_{i}\right)+ \\
& +\sum_{i=0}^{r} e^{-A_{p}(q) h_{i}} B_{p i} u(t)-\sum_{i=0}^{r} B_{p i} u\left(t-h_{i}\right)+
\end{aligned}
$$




$$
\begin{aligned}
& +A_{p}(q) \sum_{i=0}^{r} \int_{t-h_{i}}^{t} e^{-A_{p}(q)(s-t)} e^{-A_{p}(q) h_{i}} B_{p i} u(s) d s= \\
& A_{p}(q)\left(\sum_{i=0}^{r} \int_{t-h_{i}}^{t} e^{-A_{p}(q)(s-t)} e^{-A_{p}(q) h_{i}} B_{p i} u(s) d s\right)+ \\
& +\sum_{i=0}^{r} e^{-A_{p}(q) h_{i}} B_{p i} u(t)=A_{p}(q) y(t)+ \\
& +\sum_{i=0}^{r} e^{-A_{p}(q) h_{i}} B_{p i} u(t),
\end{aligned}
$$

\section{Lemma is proved}

Remark 2: The initial value for $y(t)$ in Lemma 1 is given as

$$
\begin{aligned}
& y\left(t_{0}\right)=x\left(t_{0}\right)+ \\
& +\int_{t_{0}-h_{0}}^{t_{0}} e^{-A_{p}(q)(s-t)} e^{-A_{p}(q) h_{0}} B_{p 0} u(s) d s+ \\
& +\int_{t_{0}-h_{1}}^{t_{0}} e^{-A_{p}(q)(s-t)} e^{-A_{p}(q) h_{1}} B_{p 1} u(s) d s+\cdots+ \\
& +\int_{t_{0}-h_{r}}^{t_{0}} e^{-A_{p}(q)(s-t)} e^{-A_{p}(q) h_{r}} B_{p r} u(s) d s
\end{aligned}
$$

for an arbitrary control $u(\cdot)$. We suppose that $u(\cdot) \equiv 0$ on intervals

$\left[t_{0}-h_{0}, t_{0}\right],\left[t_{0}-h_{1}, t_{0}\right], \ldots,\left[t_{0}-h_{r}, t_{0}\right]$

It follows that

$$
y\left(t_{0}\right)=x\left(t_{0}\right)
$$

Remark 3: For $r=1$ and $h_{0}=0$ the transformation is proposed in [9]. This approach is known as a predictor-like technique.

Remark 4: The general linear system with input delay is considered in [8]. The delayed action is generated by a Lebesque - Stieltjes integration of the control.

We will now introduce the optimal LQ controller with the prescribed degree of stability $\alpha$ for fixed $p$ and

$$
\sum_{i=1}^{l} q_{p i} A_{p i}=0, q_{p i} \in Q_{1} \subset R^{l},
$$

In that case, relation (8) has the form

$$
\dot{y}(t)=A_{p 0} y(t)+\sum_{i=0}^{r} e^{-A_{p 0} h_{i}} B_{p i} u(t),
$$

The index of performance is given in [18] as

$$
J_{p}^{1}=\int_{t=t_{0}}^{\infty} e^{2 \alpha \tau}\left(y^{T}(\tau) Q y(\tau)+u^{T}(\tau) R u(\tau)\right) d \tau
$$

$\alpha>0$

where $Q$ and $R$ are positive definite matrices. The optimal controller is (for fixed $p$ )

$$
\begin{gathered}
u(t)=-R^{-1}\left(\sum_{i=0}^{r} e^{-A_{p 0} h_{i}} B_{p i}\right) P_{p}^{1} y(t) \\
\min J_{p}^{1}=e^{-2 \alpha t_{0}} y^{T}\left(t_{0}\right) P_{p}^{1} y\left(t_{0}\right),
\end{gathered}
$$

where matrix $P_{p}^{1}$ is a solution of the following algebraic Riccati equation

$$
\begin{aligned}
& P_{p}^{1}\left(A_{p 0}+\alpha I\right)+\left(A_{p 0}^{T}+\alpha I\right) P_{p}^{1}- \\
& -P_{p}^{1}\left(\sum_{i=0}^{r} e^{-A_{p 0} h_{i}} B_{p i}\right) R^{-1} P_{p}^{1}+Q=0,
\end{aligned}
$$

It is well known that for LQ controller (11)(13) the Lyapunov function

$$
V(x)=y^{T}(t) P_{p}^{1} y(t),
$$

In the presence of unmodeled dynamics, the transformed closed-loop system has the following form

$$
\begin{aligned}
& \dot{y}(t)=A_{p}(q) y(t)+\sum_{i=0}^{r} e^{-A_{p}(q) h_{i}} B_{p i} u(t), \\
& u(t)=-R^{-1}\left(\sum_{i=0}^{r} e^{-A_{p 0} h_{i}} B_{p i}\right) P_{p} y(t) \\
& p=1,2, . ., s \quad, \quad h_{0}=0,
\end{aligned}
$$

where matrix $P_{p}$ is solution of algebraic Riccati equation for system with unmodeled dynamics.

The next goal is to find matrix $P_{p}$ without the help of Riccati equation. Instead, we shall use the LMI tool. The result will be formulated in the form of theorem.

Theorem 1. Let us suppose that for the closedloop system (15), (16) is satisfied

$1^{\circ}$ The pair of matrices

$$
\left[A_{p 0}+\alpha, \sum_{i=0}^{r} e^{-A_{p 0} h_{i}} B_{p i}\right]
$$

is controllable

$2^{\circ} \quad$ The matrices $Q$ and $R$ are positive definite Then the feedback law has a form 
$u(t)=-R^{-1}\left(\sum_{i=0}^{r} e^{-A_{p 0} h_{i}} B_{p i}\right)^{T}\left(Y_{p}\left(\gamma_{p}^{*}\right)\right)^{-1} y(t)$

and the upper bound for index of performance is

$J_{p} \leq e^{-2 \alpha t_{0}} \varphi\left(\gamma_{p}^{*}\right)$

for $\forall q \in Q_{l} \subset R^{l}$ and $p=1,2, . ., s$

where for $\forall \gamma_{p}>0$

$\gamma_{p}^{*}=\arg \min \varphi\left(\gamma_{p}\right), \varphi\left(\gamma_{p}\right)=$

$=\gamma_{p}^{-1} y\left(t_{0}\right)^{T}\left(Y_{p}\left(\gamma_{p}\right)\right)^{-1} y\left(t_{0}\right)$

and $Y_{p}\left(\gamma_{p}\right)$ is a solution of the following LMI

$\left[\begin{array}{ll}C_{p} & D_{p} \\ E_{p} & F_{p}\end{array}\right] \leq 0$

$C_{p}=\left(A_{p}(q)+\alpha I\right) Y_{p}+Y_{p}\left(A_{p}(q)+\alpha I\right)^{T}+$

$\left(\gamma_{p}-2\right)\left(\sum_{i=0}^{r} e^{-A_{p 0} h_{i}} B_{p i}\right)^{T} R^{-1}\left(\sum_{i=0}^{r} e^{-A_{p 0} h_{i}} B_{p i}\right)$,

$D_{p}=\gamma_{p}^{1 / 2} Y_{p} Q^{1 / 2}, E_{p}=\gamma_{p}^{1 / 2} Q^{1 / 2} Y_{p}$,

$F_{p}=-I, Y_{p}>0, \forall q \in Q_{l} \subset R^{l}$ and

$p=1,2, . ., s$

Proof: Let us introduce the following transformation:

$\dot{\hat{y}}(t)=e^{\alpha t} y(t), \quad \hat{u}(t)=e^{\alpha t} u(t)$,

Now, from relations (15) and (17) we have

$$
\dot{\hat{y}}(t)=\left(A_{p}(q)+\alpha I\right) \hat{y}(t)+\sum_{i=0}^{r} e^{-A_{p 0} h_{i}} B_{p i} \hat{u}(t),
$$

Using $1^{\circ}$ and $2^{\circ}$ conditions of theorem we have, based on analogy with (16)

$$
\hat{u}(t)=-R^{-1}\left(\sum_{i=0}^{r} e^{-A_{p 0} h_{i}} B_{p i}\right)^{T} P_{p} \hat{y}(t),
$$

From the last two relations it follows that

$$
\begin{aligned}
& \dot{\hat{y}}(t)=\tilde{A}_{p}(q) \hat{y}(t), \tilde{A}_{p}(q)=A_{p}(q)+\alpha I- \\
& -\left(\sum_{i=0}^{r} e^{-A_{p 0} h_{i}} B_{p i}\right) R^{-1}\left(\sum_{i=0}^{r} e^{-A_{p 0} h_{i}} B_{p i}\right)^{T} P_{p},
\end{aligned}
$$

Using observation (14) we have

$\dot{V}=\frac{d}{d t} V(\hat{y}(t))=$

$=\hat{y}^{T}(t)\left[\tilde{A}_{p}^{T}(q) P_{p}+P_{p} \tilde{A}_{p}(q)\right] \hat{y}(t)$,

Condition $\dot{V}<0$ will be satisfied if

$\tilde{A}_{p}^{T}(q) P_{p}+P_{p} \tilde{A}_{p}(q)<0$,

From (20) and (22) we have

$$
\begin{aligned}
& \left(A_{p}(q)+\alpha I\right)^{T} P_{p}+P_{p}\left(A_{p}(q)+\alpha I\right)- \\
& -2 P_{p}\left(\sum_{i=0}^{r} e^{-A_{p 0} h_{i}} B_{p i}\right) . \\
& R^{-1}\left(\sum_{i=0}^{r} e^{-A_{p 0} h_{i}} B_{p i}\right)^{T} P_{p}<0,
\end{aligned}
$$

Let us multiply the last inequality from the left and right side with the matrix $Y_{p}=P_{p}^{-1}$. It follows that, for $Y_{p}>0$

$$
\begin{aligned}
& \left(A_{p}(q)+\alpha I\right)^{T} Y_{p}+Y_{p}\left(A_{p}(q)+\alpha I\right)- \\
& -2\left(\sum_{i=0}^{r} e^{-A_{p 0} h_{i}} B_{p i}\right) R^{-1}\left(\sum_{i=0}^{r} e^{-A_{p 0} h_{i}} B_{p i}\right)^{T}<0,
\end{aligned}
$$

The matrix $Y_{p}$ is the solution of the inequality (24). Having in mind that $P_{p}=Y_{p}^{-1}$, the optimal performance index is (as in relation (12))

$$
J_{p}=e^{-2 \alpha t_{0}} y^{T}\left(t_{0}\right) Y_{p}^{-1} y\left(t_{0}\right)
$$

But, owing to the presence of unmodeled dynamic, $J_{p}$ cannot be determined a priori and, as a result, it cannot be used for supervisor design. But, it is possible to determine the upper bound for $J_{p}$. For that purpose, it will be considered a stronger inequality in comparison to (24).

Let us introduce the parameter $\gamma_{p}>0$ and from (24) we have 


$$
\begin{aligned}
& \left(A_{p}(q)+\alpha I\right)^{T} Y_{p}+Y_{p}\left(A_{p}(q)+\alpha I\right) \\
& -2\left(\sum_{i=0}^{r} e^{-A_{p 0} h_{i}} B_{p i}\right) R^{-1}\left(\sum_{i=0}^{r} e^{-A_{p 0} h_{i}} B_{p i}\right)^{T} \\
& +\gamma_{p} \cdot\left[\left(\sum_{i=0}^{r} e^{-A_{p 0} h_{i}} B_{p i}\right) R^{-1}\left(\sum_{i=0}^{r} e^{-A_{p 0} h_{i}} B_{p i}\right)^{T}+\right. \\
& \left.+Y_{p} Q Y_{p}\right] \leq 0 \quad, \quad Y_{p}>0,
\end{aligned}
$$

Now we shall multiply the last inequality from the left and right-hand sides with the matrix $Y_{p}^{-1}$. It follows that

$$
\begin{aligned}
& \left(A_{p}(q)+\alpha I\right)^{T} Y_{p}^{-1}+Y_{p}^{-1}\left(A_{p}(q)+\alpha I\right)-2 Y_{p}^{-1} \\
& \left(\sum_{i=0}^{r} e^{-A_{p 0} h_{i}} B_{p i}\right) R^{-1}\left(\sum_{i=0}^{r} e^{-A_{p 0} h_{i}} B_{p i}\right)^{T} \\
& Y_{p}^{-1} \leq-\gamma_{p}\left[Y_{p}^{-1}\left(\sum_{i=0}^{r} e^{-A_{p 0} h_{i}} B_{p i}\right) R^{-1} .\right. \\
& \left.\cdot\left(\sum_{i=0}^{r} e^{-A_{p 0} h_{i}} B_{p i}\right)^{T} Y_{p}^{-1}+Q\right]
\end{aligned}
$$

The last relation can be rewritten in the following form

$$
\begin{gathered}
\tilde{A}_{p}^{T}(q)\left(\frac{1}{\gamma_{p}} Y_{p}^{-1}\right)+\left(\frac{1}{\gamma_{p}} Y_{p}^{-1}\right) \tilde{A}_{p}(q) \leq-Q- \\
-Y_{p}^{-1}\left(\sum_{i=0}^{r} e^{-A_{p 0} h_{i}} B_{p i}\right) R^{-1}\left(\sum_{i=0}^{r} e^{-A_{p 0} h_{i}} B_{p i}\right)^{T} Y_{p}^{-1},
\end{gathered}
$$

Let us put

$$
\begin{aligned}
& A=\tilde{A}_{p}(q)=A_{p}(q)+\alpha I- \\
& \left(\sum_{i=0}^{r} e^{-A_{p 0} h_{i}} B_{p i}\right) R^{-1}\left(\sum_{i=0}^{r} e^{-A_{p 0} h_{i}} B_{p i}\right)^{T} Y_{p}^{-1}, \\
& U=Q+Y_{p}^{-1}\left(\sum_{i=0}^{r} e^{-A_{p 0} h_{i}} B_{p i}\right) . \\
& \cdot R^{-1}\left(\sum_{i=0}^{r} e^{-A_{p 0} h_{i}} B_{p i}\right)^{T} Y_{p}^{-1},
\end{aligned}
$$

From [15, Theorem 3, p. 142] it follows that

$$
\begin{aligned}
& \tilde{A}_{p}^{T}(q) Z_{p}+Z_{p} \tilde{A}_{p}(q)= \\
& -\left[Q+Y_{p}^{-1}\left(\sum_{i=0}^{r} e^{-A_{p 0} h_{i}} B_{p i}\right) R^{-1} .\right. \\
& \left.\cdot\left(\sum_{i=0}^{r} e^{-A_{p 0} h_{i}} B_{p i}\right)^{T} Y_{p}^{-1}\right]
\end{aligned}
$$

and

$$
J_{p}=e^{-2 \alpha t_{0}} y\left(t_{0}\right)^{T} Z_{p} y\left(t_{0}\right),
$$

If we subtract relation (30) from relation (27) we have

$$
\begin{aligned}
& \tilde{A}_{p}^{T}(q)\left(\frac{1}{\gamma_{p}} Y_{p}^{-1}-Z_{p}\right)+ \\
& +\left(\frac{1}{\gamma_{p}} Y_{p}^{-1}-Z_{p}\right) \tilde{A}_{p}^{T}(q) \leq 0,
\end{aligned}
$$

By using [16, Proposition 4.4, p.140] one can conclude

$\frac{Y_{p}^{-1}}{\gamma_{p}}-Z_{p} \geq 0$ and $\frac{Y_{p}^{-1}}{\gamma_{p}} \geq Z_{p}$,

From (31) and (33) one can get

$$
J_{p} \leq \frac{1}{\gamma_{p}} e^{-2 \alpha t_{0}} y^{T}\left(t_{0}\right) Y_{p}^{-1} y\left(t_{0}\right),
$$

Inequality (25) can be rewritten in the following form

$$
\begin{aligned}
& \left(A_{p}(q)+\alpha I\right)^{T} Y_{p}+Y_{p}\left(A_{p}(q)+\alpha I\right)+\left(\gamma_{p}-2\right) . \\
& \cdot\left(\sum_{i=0}^{r} e^{-A_{p 0} h_{i}} B_{p i}\right) R^{-1}\left(\sum_{i=0}^{r} e^{-A_{p 0} h_{i}} B_{p i}\right)^{T}+ \\
& +\left(\gamma_{p}^{1 / 2} Y_{p} Q^{1 / 2}\right)\left(\gamma_{p}^{1 / 2} Q^{1 / 2} Y_{p}\right) \leq 0, \quad Y_{p}>0,
\end{aligned}
$$

By using [16, Theorem 1.10, p.46] last inequality will take the form

$\left[\begin{array}{ll}C_{p} & D_{p} \\ E_{p} & F_{p}\end{array}\right] \leq 0$,

where $C_{p}, D_{p}, E_{p}$ and $F_{p}$ are defined in the formulation of Theorem 1.

From inequality (36) one can find solution $Y_{p}\left(\gamma_{p}\right)$. After that, we can construct the function

$$
\varphi\left(\gamma_{p}\right)=\gamma_{p}^{-1} y^{T}\left(t_{0}\right) Y_{p}^{-1}\left(\gamma_{p}\right) y\left(t_{0}\right),
$$


From the last relation it follows that

$\gamma_{p}^{*}=\underset{\gamma_{p}}{\arg \min } \varphi\left(\gamma_{p}\right)$,

Now feedback law has the form

$$
u(t)=-R^{-1}\left(\sum_{i=0}^{r} e^{-A_{p 0} h_{i}} B_{p i}\right)^{T}\left(Y_{p}\left(\gamma_{p}^{*}\right)\right)^{-1} y(t),
$$

and the upper bound for index of performance is

$$
J_{p} \leq e^{-2 \alpha t_{0}} \varphi\left(\gamma_{p}^{*}\right) \text {, }
$$

Theorem is proved

Remark 5: If $A_{p}(q)$ is the affine matrix family and $q \in Q_{l}$ is a polygon it is enough to solve matrix inequalities (36) for vertices $\mathrm{V}$ of set $Q_{l}$. It means that we must to solve the finite number of matrix inequalities

$$
\begin{aligned}
& {\left[\begin{array}{ll}
C_{p} & D_{p} \\
E_{p} & F_{p}
\end{array}\right] \leq 0} \\
& C_{p}=\left(A_{p}\left(q^{v}\right)+\alpha I\right)^{T} Y_{p}+Y_{p}\left(A_{p}\left(q^{v}\right)+\alpha \mathrm{I}\right)^{T} \\
& +\left(\gamma_{p}-2\right) . \\
& \cdot\left(\sum_{i=0}^{r} e^{-A_{p 0} h_{i}} B_{p i}\right)^{T} R^{-1}\left(\sum_{i=0}^{r} e^{-A_{p 0} h_{i}} B_{p i}\right) \\
& D_{p}=\gamma_{p}^{1 / 2} Y_{p} Q^{1 / 2}, \mathrm{E}_{p}=\gamma_{p}^{1 / 2} Q^{1 / 2} Y_{p}, \\
& F_{p}=-I, Y_{p}>0, q^{v} \in V
\end{aligned}
$$

The matrices $A_{p}\left(q^{v}\right)$ are matrices for which $\left|q_{p i}\right|<\gamma_{p} \quad, \quad p=1,2, . ., s \quad, \quad i=1,2, . ., l$

For situation when the number of matrix inequalities is large special iterative procedures are developed in the literature.

\section{Robust Stability of Switching Control Systems}

By using relation (15) and the fact that $h_{0}=0$, the system can be described in the form

$$
\begin{aligned}
& \dot{y}(t)=A_{p 0} y(t)+B_{p 0} u(t)+\sum_{i=1}^{l} q_{p i} A_{p i} y(t) \\
& +\sum_{i=1}^{r} e^{-A_{p}(q) h_{i}} B_{p i} u(t)=A_{p 0} y(t)+B_{p 0} u(t) \\
& +\Delta(y(t), p(t), u(t), q),
\end{aligned}
$$

where

$$
\begin{aligned}
& \Delta(y(t), p(t), u(t), q)= \\
& =\sum_{i=1}^{l} q_{p i} A_{p} y(t)+\sum_{i=1}^{r} e^{-A_{p}(q) h_{i}} B_{p i} u(t),
\end{aligned}
$$

whereby $q_{p} \in Q_{l} \subset R^{l}$ and $p=1,2, . ., s$.

The switching controller has two components

A) The analog feedback

$$
\begin{aligned}
& u(t)=-R^{-1}\left(\sum_{i=0}^{r} e^{-A_{p 0} h_{i}} B_{p i}\right)^{T}\left(Y_{p}\left(\gamma_{p}^{*}\right)\right)^{-1} y(t) \\
& p=1,2, . ., s,
\end{aligned}
$$

whereby $Y_{p}\left(\gamma_{p}^{*}\right)$ is the solution of LMI which is defined in Theorem 1.

B) The discrete feedback is

$$
\begin{gathered}
p_{1}=\min \left(\varphi\left(\gamma_{p}^{*}\right)\right), p=1,2, . ., s \\
\varphi\left(\gamma_{p^{*}}\right)=\gamma_{p^{*}}^{-1} y^{T}(t)\left(Y_{p}\left(\gamma_{p^{*}}\right)\right)^{-1} y(t),
\end{gathered}
$$

Now we shall formulate a theorem which will proved robust stability of the original system (2).

Theorem 2. Suppose that for the dynamic switching system (41)-(44) the following is satisfied

$1^{\circ}$ The next two inequalities are satisfied

$$
\begin{aligned}
& \left\|\left(A_{p 0}-B_{p 0} R^{-1}\left(\sum_{i=0}^{r} e^{-A_{p 0} h_{i}} B_{p i}\right)^{T}\left(Y_{p}\left(\gamma_{p}^{*}\right)\right)^{-1}\right) y(t)\right\| \\
& \leq k_{p 1} e^{2 \alpha t}(y(t) Q y(t)+u(t) R u(t))+c \\
& \left\|\left(A_{p 0}-B_{p 0} R^{-1}\left(\sum_{i=0}^{r} e^{-A_{p 0} h_{i}} B_{p i}\right)^{T}\left(Y_{p}\left(\gamma_{p}^{*}\right)\right)^{-1}\right) y(t)\right\| \\
& \quad \geq 1 \\
& \gamma_{p}^{*}=\underset{\gamma_{p}}{\arg \min } \gamma_{p}^{-1} y^{T}(t)\left(Y\left(\gamma_{p}\right)\right)^{-1} y(t)
\end{aligned}
$$

$$
\begin{gathered}
k_{p 1}>0, c>0, p=1,2, . ., s \\
2^{\circ}\|y(t)\| \leq k_{2 p} e^{2 \alpha t}\left(y^{T}(t) Q y(t)+u^{T}(t) R u(t)\right) \\
k_{2 p}>0,\|y(t)\| \geq 1, p=1,2, . ., s
\end{gathered}
$$

$3^{\circ}$ The affine matrix family is 


$$
\begin{aligned}
& A_{p}(q)=A_{p 0}+\sum_{i=1}^{l} q_{p i} A_{p i} \\
& p=1,2, . ., s
\end{aligned}
$$

the set $Q_{l} \subset R^{l}$ is a polygon and the set of vertices $q^{v} \in V \subset Q_{l}$ is

$V=\left\{q \in Q_{l}:\left|q_{p i}\right|=\gamma_{p}^{*}\right\}$

$4^{\circ} \quad r_{\Delta} \in[0, \infty)$

where

$$
\begin{aligned}
& r_{\Delta}=\sup _{q \in Q_{1}, p \in M}\left\|\Delta_{p}(y(t), p(t), u(t), q)\right\| \\
& M=\{1,2, \ldots, s\}
\end{aligned}
$$

$5^{\circ}$ The system without unmodeled dynamics is controllable

$$
\left[A_{p 0}+\alpha, \sum_{i=0}^{r} e^{-A_{p 0} h_{i}} B_{p i}\right]
$$

$6^{\circ}$ The best performance achievable by using LQ non-switching control strategy is

$$
\begin{aligned}
& \eta_{0}=\min \left\{\frac{1}{\gamma_{p}^{*}} e^{-2 \alpha t_{0}} y^{T}\left(t_{0}\right) Y_{p}^{-1}\left(\gamma_{p}^{*}\right) y\left(\mathrm{t}_{0}\right)\right\} \\
& p=1,2, . ., s
\end{aligned}
$$

Then the completely controllable system (2) is stabilized by the control law

$u(t)=$

$$
=-K_{p}\left(x(t)+\sum_{i=1}^{r} \int_{t-h_{i}}^{t} e^{-A_{p}\left(q^{v}\right) h_{i}} e^{-A_{p}(q) h_{i}} B_{p i} u(s) d s\right)
$$$$
K_{p}=R^{-1}\left(\sum_{i=0}^{r} e^{-A_{p 0} h_{i}} B_{p i}\right)^{T}\left(Y_{p}\left(\gamma_{p}^{*}\right)\right)^{-1}
$$

is a feedback gain matrix

Proof: We shall first prove the boundedness of index performance for a switched system. Let us define the discrete state sequence

$$
p=\left\{p\left(t_{j}\right), j=0,1, \ldots .\right\} \text {, }
$$

For any integer $n>0$, the truncated switching sequence is

$$
p_{n}=\left\{p\left(t_{0}\right), p\left(t_{1}\right), . ., p\left(t_{n}\right)\right\},
$$

$$
T(t)=y^{T}(t) Q y(t)+u^{T}(t) R u(t),
$$

Now, for the performance index we have

$$
\begin{aligned}
& J_{n}=\int_{t_{0}}^{t_{1}} e^{2 \alpha t} T(t) d t+. .+\int_{t_{n-1}}^{t_{n}} e^{2 \alpha t} T(t) d t+ \\
& \quad+\int_{t_{n}}^{\infty} e^{2 \alpha t} T(t) d t= \\
& =\int_{t_{0}}^{t_{1}} e^{2 \alpha t} T(t) d t+. .+\int_{t_{n-1}}^{t_{n}} e^{2 \alpha t} T(t) d t+J_{p\left(t_{n}\right)} \leq \\
& \leq \int_{t_{0}}^{t_{1}} e^{2 \alpha t} T(t) d t+. .+\int_{t_{n-1}}^{t_{n}} e^{2 \alpha t} T(t) d t+ \\
& +\frac{1}{\gamma_{p\left(t_{n}\right)}^{*}} e^{-2 \alpha t_{n}} y^{T}\left(t_{n}\right) Y_{p\left(t_{n}\right)}^{-1}\left(\gamma_{p\left(t_{n}\right)}^{*}\right) y\left(t_{n}\right),
\end{aligned}
$$

From switching criterion (44) it follows that

$$
\begin{aligned}
& J_{n} \leq \int_{t_{0}}^{t_{1}} e^{2 \alpha t} T(t) d t+. .+\int_{t_{n-1}}^{t_{n}} e^{2 \alpha t} T(t) d t+ \\
& \frac{1}{\left.\gamma_{p\left(t_{n-1}\right)}^{*}\right)} e^{-2 \alpha t_{n-1}} y^{T}\left(t_{n-1}\right) Y_{p\left(t_{n-1}\right)}^{-1}\left(\gamma_{p\left(t_{n-1}\right)}^{*}\right) y\left(t_{n-1}\right),
\end{aligned}
$$

By using same procedure we finally have

$$
J_{n} \leq \frac{1}{\gamma_{p\left(t_{0}\right)}^{*}} y^{T}\left(t_{0}\right) Y_{p\left(t_{0}\right)}^{-1}\left(\gamma_{p\left(t_{0}\right)}^{*}\right) y\left(t_{0}\right)=\eta_{0},
$$

noting that $p\left(t_{0}\right)$ is determined according to (44). Since the right-hand side of (50) is independent of $n$, it follows that for the resultant performance index $\mathrm{J}$ of the switching systems

$$
J=\lim _{n \rightarrow \infty} J_{n} \leq \eta_{0},
$$

From relation (41) for any $\tau \in[t, t+1]$ we obtain

$$
\begin{aligned}
& y(t)=y(\tau)- \\
& -\int_{t}^{\tau}\left(A_{p 0}-B_{p 0} R^{-1}\left(\sum_{i=0}^{r} e^{-A_{p 0} h_{i}} B_{p i}\right)^{T}\left(Y_{p}\left(\gamma_{p}^{*}\right)\right)^{-1}\right) . \\
& y(\theta) d \theta+\int_{t}^{\tau} \Delta_{p}(y(\theta), p(\theta), u(\theta), q) d \theta,
\end{aligned}
$$

The last relation yields

Let us introduce 


$$
\begin{aligned}
& \|y(t)\| \leq\|y(\tau)\|+ \\
& \int_{t}^{\tau}\left\|\left[A_{p 0}-B_{p 0} R^{-1}\left(\sum_{i=0}^{r} e^{-A_{p 0} h_{i}} B_{p i}\right)^{T}\left(Y_{p}\left(\gamma_{p}^{*}\right)\right)^{-1} y(\theta)\right]\right\| d \theta \\
& +\int_{t}^{\tau}\left\|\Delta_{p}(y(\theta), p(\theta), u(\theta), q)\right\| d \theta,
\end{aligned}
$$

Let us introduce the following sets

$$
\Omega=\{\theta \in[t, t+1]\},
$$

and

$$
\Omega_{1}=\{\theta \in[t, t+1]:
$$$$
\left.\left\|A_{p 0}-B_{p 0} R^{-1}\left(\sum_{i=0}^{r} e^{-A_{p 0} h_{i}} B_{p i}\right)^{T}\left(Y_{p}\left(\gamma_{p}^{*}\right)\right)^{-1} y(\theta)\right\|<1\right\},
$$

Now we will define the next set

$$
\Omega_{2}=\Omega \backslash \Omega_{1},
$$

From (51), (53) and condition $1^{\circ}$ of theorem it follows that

$$
\begin{aligned}
& \|y(t)\| \leq\|y(\tau)\|+\int_{\Omega_{1}} d \theta+ \\
& \int_{\Omega_{2}}\left(k_{p 1} e^{2 \alpha \theta}\left(y^{T}(\theta) Q y(\theta)+u^{T}(\theta) R u(\theta)\right)+c\right) d \theta+ \\
& +\int_{\Omega}\left\|\Delta_{p}(y(\theta), p(\theta), u(\theta), q)\right\| d \theta \\
& \leq\|y(\tau)\|+1+c+k_{p 1} J(t)+r_{\Delta} \leq \\
& \leq\|y(\tau)\|+1+c+k_{p 1} \eta+r_{\Delta},
\end{aligned}
$$

Condition $2^{\circ}$ of the theorem implies for $\forall y(t)$

$\|y(\tau)\| \leq$

$k_{p 2} e^{2 \alpha t}\left(y^{T}(t) Q y(t)+u^{T}(t) R u(t)\right)+1$,

Relations (57) and (58) together give

$$
\begin{aligned}
& \|y(t)\| \leq \int_{t}^{t+1}\left(\|y(\theta)\|+1+c+k_{p 1} r+r_{\Delta}\right) d \theta \leq \\
& \leq \int_{t}^{t+1}\left(k_{p 2} e^{2 \alpha \theta}\left(y^{T}(\theta) Q y(\theta)+u^{T}(\theta) R u(\theta)\right)+1\right) d \theta+ \\
& +1+c+k_{p 1} \eta+r_{\Delta} \leq \\
& \leq k_{p 2} \int_{t}^{t+1} e^{2 \alpha \theta}\left(\hat{y}^{T}(\theta) Q \hat{y}(\theta)+\hat{u}^{T}(\theta) R \hat{u}(\theta)\right) d \theta+ \\
& +2+c+k_{p 1} \eta+r_{\Delta} \leq k_{p 2} \eta+2+c+k_{p 1} \eta+r_{\Delta}= \\
& =\left(k_{p 2}+k_{p 1}\right) \eta+2+c+r_{\Delta},
\end{aligned}
$$

Let us introduce

$$
\begin{aligned}
& k=\max _{p}\left(k_{p 1}+k_{p 2}\right) \\
& p=1,2, . ., s,
\end{aligned}
$$

From (59) and (60) it fallows that

$$
\|y(t)\|_{\infty} \leq k \eta+2+c+r_{\Delta},
$$

Since the right-hand side is independent of $t$

$\|y(t)\|_{\infty} \leq \infty$

$p=1,2, \ldots, s$,

The transformation relation has the compact form

$$
\begin{aligned}
& y(t)=x(t)+ \\
& \sum_{i=1}^{r} \int_{t-h_{i}}^{t} e^{-A_{p}(q)(s-t)} e^{-A_{p}(q) h_{i}} B_{p i} u(s) d s,
\end{aligned}
$$

From the last relation we obtain

$$
\begin{aligned}
& \|x(t)\| \leq\|y(t)\|+\sum_{i=1}^{r} h_{i} \max _{-h_{i} \leq \theta \leq 0}\left\|e^{A_{p}(q) \theta}\right\| . \\
& \cdot\left\|B_{p i}\right\|\|K\|\|y(t)\|,
\end{aligned}
$$

From relation (62) and (64) it follows that

$\|x(t)\|_{\infty}<\infty$

This concludes the proof of Theorem.

Remark 6: Here we shall comment about the conditions $1^{\circ}$ and $2^{\circ}$ of Theorem 2 . Let us consider the analog nonlinear system in state space form

$$
\dot{y}(t)=f(y(t), t),
$$


where $y(t) \in R^{n}$ is state variable. Let us further suppose that a performance measure for the system.

$$
J(t)=\int_{t}^{\infty} h(y(\tau), \tau) d \tau \quad, \quad h(y(\tau), \tau) \geq 0,
$$

In reference [19], the performance dominant conditions are defined. The performance measure $J(t)$ is said to be dominant for the system (66) if there exist constants $c, k_{1}, k_{2} \geq 0$ such that for all $t \geq 0$

$$
\begin{aligned}
& \|f(y(t), t)\| \leq k_{1} h(y(t), t)+c, \\
& \text { for }\|f(y(t), t)\| \geq 1 \\
& \|y(t)\| \leq k_{2} h(y(t), t), \text { for }\|(y(t))\| \geq 1,
\end{aligned}
$$

Relation (68) means that only finite escaping of stales will show up in the performance measure $J(t)$. Relation (69) means that large persistent $y(t)$ values must be detected in the index of performance avoiding the situation in which the tail of $y(t)$ remains large even when the index of performance $J(t)$ is bounded. Using relation (68) and (69), we can easily get the assumptions $1^{\circ}$ and $2^{\circ}$ of Theorem 2 .

Remark 7: Here we shortly consider design of switching controllers for systems with output delay. The system is described as

$$
\begin{aligned}
& \dot{x}(t)=A_{p}(q) x(t)+B_{p} u(t), \\
& \bar{z}(t)=\bar{C}_{p} x(t-h), \\
& p=1,2, . ., s
\end{aligned}
$$

In relation (70) $\mathrm{h}$ is the output time delay. Now we introduce transformation (that is a special case of Lemma 1) for the system (70) and (71).

$$
\begin{gathered}
z(t)=\bar{z}(t)+\bar{C}_{p} e^{-A_{p}(q) h} . \\
\int_{t-h}^{t} e^{-A_{p}(q)(t-h)} B u(\tau) d \tau,
\end{gathered}
$$

The transformed system has the form

$$
\begin{aligned}
& \dot{x}(t)=A_{p}(q) x(t)+B_{p} u(t), \\
& z(t)=C_{p} x(t), C_{p}=\bar{C}_{p} e^{-A_{p}(q) h},
\end{aligned}
$$

By using LMI methodology we can get controller
$u(t)=-K_{p} z(t)=-K_{p} C_{p} x(t)$,

The supervisor (multi - estimator, monitoring signal generator, and switching logic) has the form as in the reference [2]. For the stability analysis of switching system (73)-(75) it is possible to use Theorem 2.

From the short consideration it follows that stability analysis for switching systems with input delay and switching systems with output delay is the same.

\section{Conclusion}

This paper considers the problem of design of switching LQ contrrollers for systems with input time-delay. The problem is solved using suitable transformation which converts original time-delay systems into the form of delay free systems. The paper uses the concept of multiple models and switching controllers. The switching strategy is determined by using upper bounds for indices of performance. For switching systems the asymptotic sability in the sense of $\infty-$ norm is proved. Further investigation is required for the situation when the matrix $\mathrm{B}$, also, has uncertainty.

\section{REFERENCES}

1. TABUDA, P., Verification and Control of Hybrid Systems. A Symbolic Approach, Springer, Berlin, 2009.

2. LIBERZON, D., Switching in Systems and Control, Springer, Berlin, 2003.

3. WANG, H. P, C. VASSEUR, V. CONCAR, A. CHAMROO, N. CHRISTOV, Design and Implementation of Robust Hybrid Control of Vision Based Underactuated Mechanical Nonminimum Phase Systems, Studies in Informatics and Control, Vol. 19(1), 2010, pp. 35-44.

4. FILIPOVIC, V. Z., Global Exponential Stability of Switched Systems, Applied Mathematics and Mechanics-English Edition, Vol.32(9), 2011, pp. 1197-1206.

5. SOLTANPOUR, M., S. SHAFIE, Design and Stability Analysis of a Robust Impedance Control Systems for a Robust Manipulator, Studies in Informatics and Control, Vol.19(1), 2010, pp. 15-16. 
6. NICULESCU, S.-I., Delay Effects on Stability. A Robust Control Approach, Springer. Berlin, 2001.

7. YANUSHEVSKY, R. T., Control and Time-Delay Systems (in Russian), Nauka, Moscow, 1978.

8. ARSTEIN, Z., Linear Systems with Delayed Controls: a Reduction, IEEE Transaction on Automatic Control, Vol. 27(8), 1982, pp. 869-879.

9. KWON, W. H., A. E. PEARSON, Feedback Stabilization of Linear Systems with Delayed Control, IEEE Transaction on Automatic Control, Vol. 25(2), 1980, pp.266-269.

10. WANG, H., V. KONCAR, N. CHRISTOV, C. VASSEUR, A. CHAMROO, Sampled Tracking for Delayed Systems Using Two-Time-Scale Sampled- data Controllers, Studies in Informatics and Control, Vol. 19(4), 2010, pp. 339-346.

11. FILIPOVIC, V. Z, Switching Control in the Presence of Constraints and Unmodeled Dynamics, in: New Approach in Automation and Robotics, Ed. H. Aschemann, I Tech, Vienna, 2008, pp. 225-238.

12. FILIPOVIC, V. Z, Exponential Stability of Stochastic Switched Systems, Transaction of the Institute of Measurement and Control, Vol. 31(2), 2009, pp. 205-212.
13. BOYD, S., L. EL GHAOUI, E. FERON, V. BALAKRISHNAN, Linear Matrix Inequalities in Systems and Control Theory, SIAM, Philadelphia, 1994.

14. HESPANAHA, J., D. LIBERZON, A. S. MORSE, B. D. O. ANDERSON, T. S.BRINSMEAD, F. DE BRUYNE, Multiple Model Adaptive Control. Part 2: Switching. International Journal on Robust and Nonlinear Control, Vol. 11(5), 2001, pp. 479-496.

15. ANDREEV, YU. N., Control of Finite Dimensional Control Systems (in Russian), Nauka, Moscow, 1976.

16. DULlerud, G. E., F. PAGANINI, A Course in Robust Control Theory. A Convex Approach, Springer, Berlin, 2000.

17. POLYAK, B. T., P. S. SCHERBAKOV, Robust Stability and Control (in Russian), Nauka, Moscow 2002.

18. ANDERSON, B. D. O., J. B. MOORE, Optimal Control and Linear Quadratic Methods, Prentice-Hall, New Jersey, 1989.

19. WANG, L. Y., P. KHARGONEKAR, A. BEYDOUN, Robust Control of Hybrid Systems: Performance Guided Strategies, in: Hybrid systems V, Eds. P. Antsaklis, W. Kohn, M. Lemmon, A. Nerode and S. Sastry, Springer, Berlin, 1999, pp. 356-389. 